\title{
Supporting Information: Inducing and Controlling Molecular Magnetism through Supramolecular Manipulation
}

Jan Homberg, Alexander Weismann, ${ }^{*}$ Richard Berndt, and

Manuel Gruber*

Institut für Experimentelle und Angewandte Physik,

Christian-Albrechts-Universität, 24098 Kiel, Germany

E-mail: weismann@physik.uni-kiel.de; gruber@physik.uni-kiel.de 


\section{Contents}

$\begin{array}{ll}\text { I. Supporting experimental data } & \text { S-3 }\end{array}$

A. Details on supramolecules . . . . . . . . . . . . . . . . S-3

B. Supplementary topographs . . . . . . . . . . . . . . . . . S-4

C. Influence of the tautomerization of the central molecule . . . . . . . . . . S-5

D. Identification of orbitals . . . . . . . . . . . . . . . . . . . S-6

1. Single $H_{2} P c$ molecule . . . . . . . . . . . . . . . . . S-6

2. Supramolecule . . . . . . . . . . . . . . . S . . . . .

E. YSR resonances for additional tautomer configurations . . . . . . . . . . . S-8

II. Density functional theory related modeling $\quad$ S-10

A. DFT calculations . . . . . . . . . . . . . . . . . . . . . . . . . .

B. Simulation of $\mathrm{dI} / \mathrm{dV}$ maps . . . . . . . . . . . . . . . . . . S 1 . . . . . . . . . . . .

C. Effective tight binding Hamiltonian from DFT . . . . . . . . . . . . . . S-11

D. Phenomenological intermolecular interaction model . . . . . . . . . . . . . S-13

1. Description of the model . . . . . . . . . . . . . S-13

2. Nearest neighbor interaction . . . . . . . . . . . . . S-16

3. Electrostatic interaction from unperturbed $\mathrm{H}_{2} \mathrm{Pc}$ molecules . . . . . . . S S-16

4. Electrostatic interaction due to charge redistribution . . . . . . . . . S-17

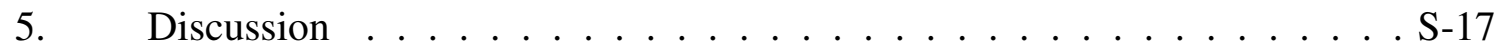

$\begin{array}{lr}\text { References } & \text { S-20 }\end{array}$ 


\section{Supporting experimental data}

\section{A. Details on supramolecules}

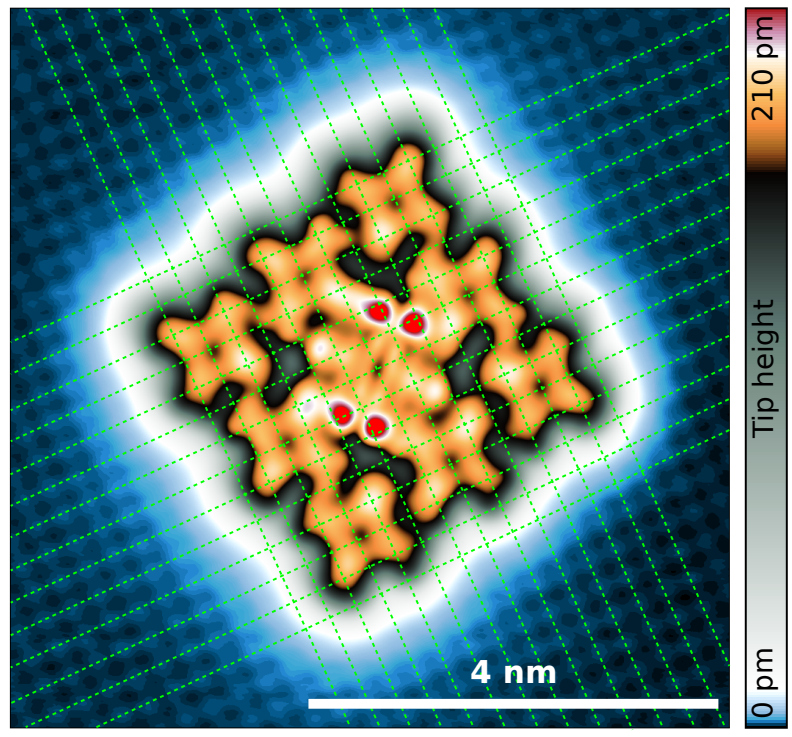

Figure S1: Epitaxy of the molecules within a cluster on a $\mathrm{Pb}(100)$ substrate. Constant-current topograph of a supramolecule A measured at $V=-10 \mathrm{mV}$ and $I=500 \mathrm{pA}$. Dashed green lines interpolate the $\mathrm{Pb}$ lattice that is resolved around the cluster. The centers of all molecules are located atop $\mathrm{Pb}$ atoms.

Figure S1 shows a high-resolution STM topograph of a supramolecule A along with atomic resolution of the $\mathrm{Pb}(100)$ surface. The intersections between the dashed green lines indicate the positions of the surface $\mathrm{Pb}$ atoms. Within the uncertainty of the experiment, the centers of all molecules are located atop $\mathrm{Pb}$ atoms. We assume that the commensurability between the corner molecules and the $\mathrm{Pb}(100)$ lattice observed in Figure $\mathrm{S} 1$ is preserved for all configurations $\mathrm{A}-\mathrm{D}$ and $\mathrm{A}^{*}-\mathrm{D}^{*}$. The systems comprising a supramolecule and the $\mathrm{Pb}(100)$ substrate are chiral. Below, however, we do not distinguish between the enantiomers.

$\mathrm{A}^{*}$ is the least stable configuration investigated. In contrast to the other configurations, we were not able to directly construct a cluster $A^{*}$ through the assembly of nine molecules. Instead, we first built a cluster $\mathrm{A}$, added three more molecules at one edge to form a $3 \times 4$ cluster and then removed three molecules from the opposite edge (Figure S2 a-c). This procedure resulted in a cluster of type $A^{*}$. However, it switched to configuration A during a subsequent acquisition of a topograph with $I=100 \mathrm{pA}$ and $V=10 \mathrm{mV}$ (Figure S2d). Besides the change in the orientations of the molecules, protrusions are visible over the lower half of the central molecule (imaged after the transition). These protrusions indicate the presence of YSR resonances in configuration A, which were presumably absent in configuration $A^{*}$. It may be worth mentioning that similar cooperative 


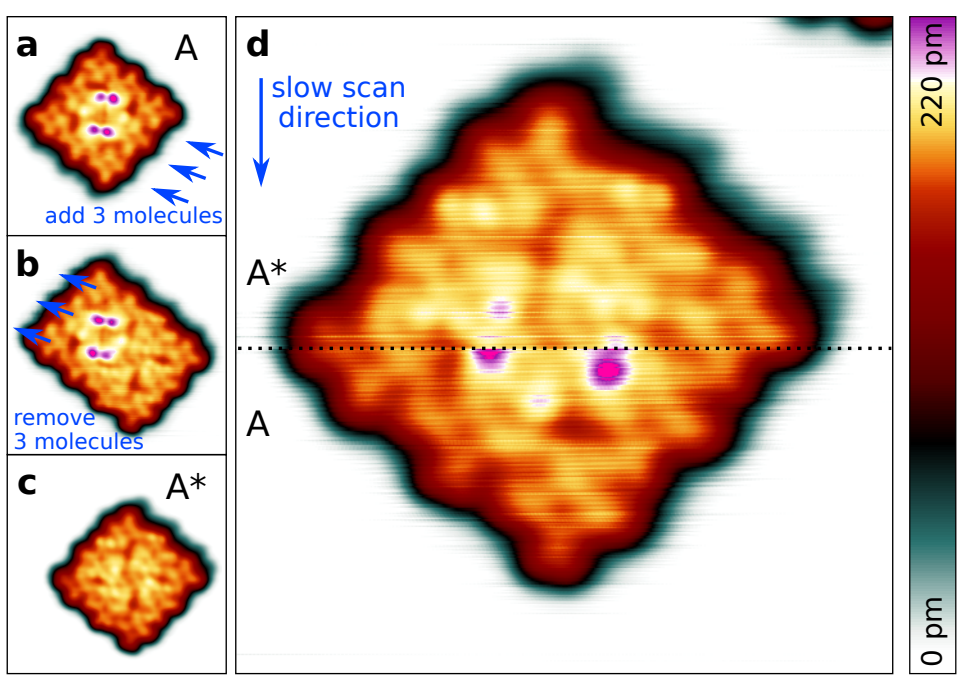

Figure S2: Assembly of a A* supramolecule. Sequence of constant-current topographs displaying some steps of the construction of a supramolecule $A^{*}$. The sequence starts from a, a cluster A to which three further molecules (arrows in $\mathbf{a}$ ) are added to produce $\mathbf{a} \mathbf{b}, 4 \times 3$ cluster. In $\mathbf{c}$, cluster $\mathrm{A}^{*}$ is obtained by removing the three upper left molecules (indicated in $\mathbf{b}$ with arrows). $\mathbf{d}$, Clusters $\mathrm{A}^{*}$ are less stable than type $\mathrm{A}$ and switch to configuration A during scanning. A dashed line in $\mathbf{d}$ separates the upper part of the topograph, acquired before the $\mathrm{A}^{*}$ to $\mathrm{A}$ transition from the lower part that was imaged after the transition. The topographs were acquired with a sample voltage of $10 \mathrm{mV}$ and tunneling currents: a-c, $50 \mathrm{pA}$ and $\mathbf{d}, 100 \mathrm{pA}$. The widths of the images are $10 \mathrm{~nm}$ for $\mathbf{a}-\mathbf{c}$ and $8 \mathrm{~nm}$ for $\mathbf{d}$.

in-plane rotational switching of multiple phthalocyanine molecules has been reported in Ref. S1. The $4 \times 3$ cluster shown in Figure S2b may be viewed as a combination of A and A* supramolecules. Indeed, such supramolecules can be obtained by removing the three top left or three bottom right molecules. In analogy, the self assembled layer of $\mathrm{H}_{2} \mathrm{Pc}$ on $\mathrm{Pb}(100)$, discussed in the main manuscript, is composed of $\mathrm{A}$ and $\mathrm{A}^{*}$ supramolecules. The molecules marked in black in Figure 3a of the main manuscript exhibit YSR resonances and are located in environments that resemble an $\mathrm{A}$ cluster. Similarly, molecules without YSR resonance (blue) are surrounded by an A*-like neighbor shell.

\section{B. Supplementary topographs}

The measurements described below have been taken on two manually assembled $\mathrm{H}_{2} \mathrm{Pc}$ type A clusters. The tautomer configurations of the molecules within the supramolecule were modified by placing the STM tip above a molecular lobe and increasing the sample voltage to $600 \mathrm{mV}$ at $I=200 \mathrm{pA}$. Tautomerization events lead to two-level fluctuations of the apparent height of the molecule. Once the tautomer of interest was obtained, the voltage was quickly reduced. This operation was repeated on each molecule of the supramolecule to obtain a desired tautomer con- 
figuration. Figures S3a-b show different configurations of two supramolecules measured with two different tips. YSR resonances were measured on each of these configurations (Figure 10 of the main text and Figure S7).

The configurations of the supramolecule are illustrated by schematics in Figure S3. Ovals correspond to the lobes of the molecules to which the inner hydrogen atoms are bonded. The molecules are colored in gray for the central molecules, in red when the axis defined by the two ovals is passing through the central molecule and in blue otherwise. As discussed below, the distinction between red and blue molecules is useful to predict the LUSO and YSR-resonance energies of the central molecule.

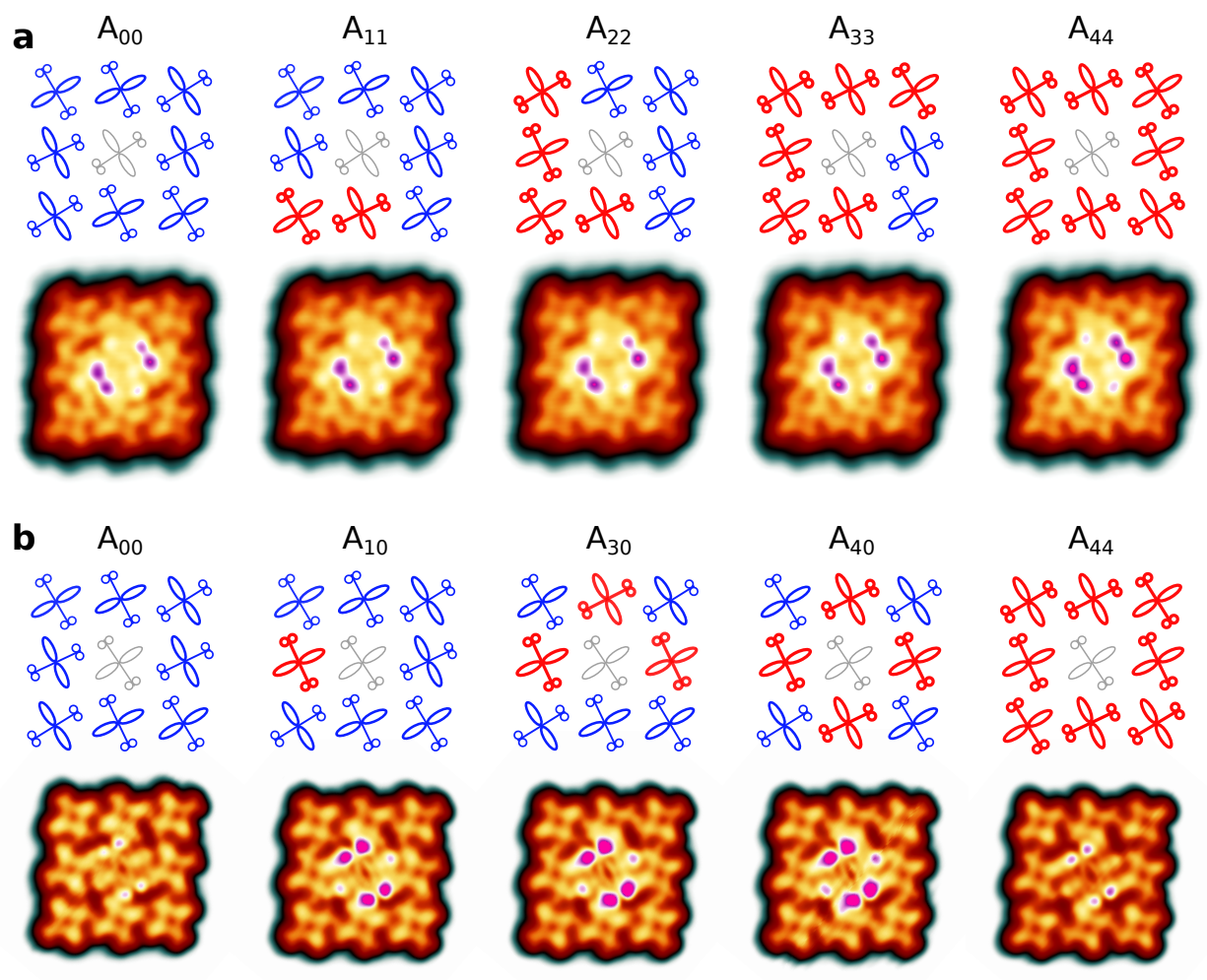

Figure S3: Different tautomer configurations realized in two supramolecules. a-b, Schematics and STM topographs ( $U=10 \mathrm{mV}, I=100 \mathrm{pA})$ of tautomer configurations. The configurations were prepared on two different supramolecules and with two different tips. The topographs of $\mathrm{A}_{00}$ and $\mathrm{A}_{44}$ in $\mathbf{b}$ have been measured at a sample voltage of $U=-10 \mathrm{mV}$. Therefore, the protrusions on the central molecules are less prominent.

\section{Influence of the tautomerization of the central molecule}

Figures S4a and b show topographs of the same supramolecule A acquired before and after tautomerization of the central molecule. The tautomerization effectively rotates the protrusions (purple) that are related to YSR resonances by $90^{\circ}$. This rotation is expected because the spatial 
distribution of YSR resonances is linked to the LUMO of the central molecule (approximately corresponding to the LUSO), which rotates upon tautomerization.

Apart from this rotation, the tautomerization of the central molecule has little effect on the YSR resonances as displayed in Figure S4c (red and blue curves). The peaks of both tautomers are closer to the Fermi level than the two coherence peaks of the substrate (dashed lines) and their heights are asymmetric.

The YSR peaks of configuration 1 are slightly $(\approx 10 \mu \mathrm{V})$ closer to the Fermi level than observed for configuration 2. We attribute this difference to the asymmetries of the LUSO and the electrostatic potential induced by the neighbors. Indeed, the rotation of the LUSO modifies the volume integral of the electrostatic potential multiplied by the LUSO density (Equation 9), which affects the LUSO energy. In turn, the LUSO energy change corresponds to an evolution of the YSR energy.

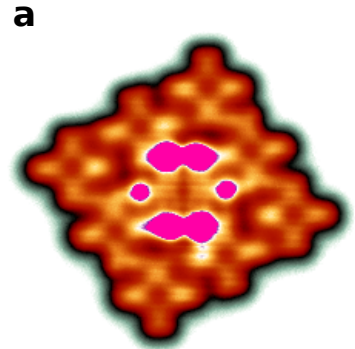

Config. 1
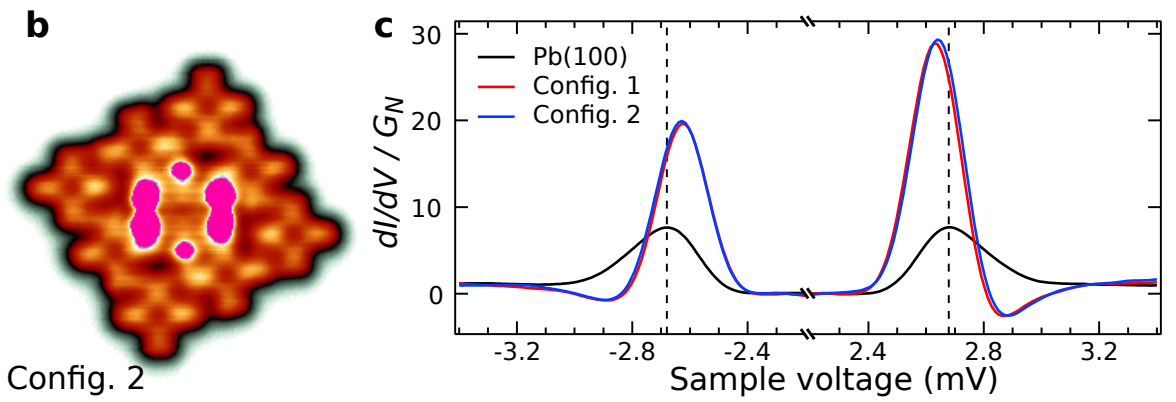

Figure S4: STM topographs ( $8 \mathrm{~nm}$ wide) of a cluster A $\mathbf{a}$ before and $\mathbf{b}$ after tautomerization of the central molecule. c, Differential conductance spectra acquired atop the central molecules (purple areas in a-b) before (red) and after (blue) its tautomerization, along with a $d I / d V$ spectrum acquired over $\mathrm{Pb}(100)$ (black) under identical conditions. All spectra are normalized to the conductance $G_{N}$ in the normal state of substrate and tip. The dashed black lines indicate the positions of the $\mathrm{Pb}$ coherence peaks.

\section{Identification of orbitals}

\section{Single $H_{2} P c$ molecule}

Figures S5a and $\mathrm{b}$ show that the peak position of the resonances at $\approx 0.2 \mathrm{~V}$ depends on the spatial location over the molecule. As an alternative to fits with two Lorentzians (main manuscript), we fitted spectra recorded on a grid with single Lorentzians and display the resulting peak positions in Figure S5c. The peak occurs at lower voltages at the lobes of the molecule and shifts upwards by up to $70 \mathrm{mV}$ at the center of the molecule. We tentatively attribute this shift to vibration assisted tunneling as described by Pavliček et al. ${ }^{\mathrm{S} 2}$ At the center of the molecule, the overlap between the LUMO and an $s$-wave tip vanishes by symmetry and the expected conductance is zero. Vibrations 

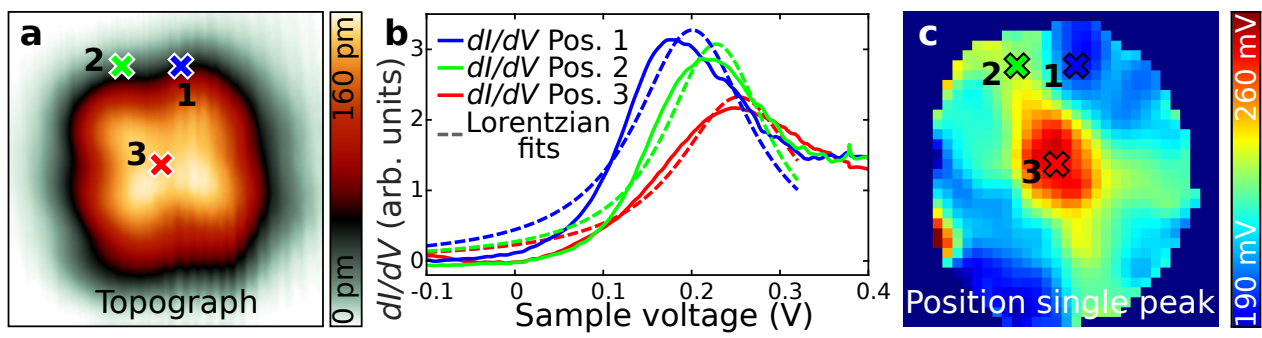

Figure S5: Spatial dependence of $d I / d V$ spectra. a, Topograph of a single $\mathrm{H}_{2} \mathrm{Pc}$ molecule. The crosses indicate the locations where $d I / d V$ spectra were recorded. b, Background subtracted $d I / d V$ spectra measured at the positions indicated in (a) along with fits using a single Lorentzian. c, Map of the peak positions obtained from the fits.

of the molecules, however, break the LUMO symmetry. The apparent shift of the orbital $(\approx 70 \mathrm{mV})$ therefore may be interpreted as the energy required to excite a molecular vibration.

\section{Supramolecule}

As described in the main manuscript, the supramolecular orbitals can be approximated by molecular orbitals of individual molecules. For instance, the spatial distribution of the LUSO is very close to that of the LUMO applied on the central molecule. The two denominations are hereafter equivalently employed.

Figure S6 shows a $d I / d V$ spectrum, measured atop the center of a supramolecule A (see inset). Similar to the case of the single molecule, the LUSO and the LUSO+1 energies are not resolved but the orbitals are spatially separated. However, the positions with maximum weight of the LUSO are close to the rim of the molecule (see purple areas in Figure S6) where the spectrum contains contributions from neighbors. In addition, the spectrum exhibits YSR resonances, which are broadened by the voltage modulation ( $2.5 \mathrm{mV}$ amplitude).

To determine the different contributions to the spectrum, we fit the data with the sum of six Lorentzians convoluted with a function that describes the broadening caused by the voltage modulation used for lock-in detection ${ }^{\mathrm{S} 3}$

$$
f_{\text {lock-in }}(V)= \begin{cases}\frac{2}{\pi V_{m}^{2}} \sqrt{V_{m}^{2}-V^{2}} & ,|V| \leq V_{m} \\ 0 & ,|V|>V_{m}\end{cases}
$$

where $V$ is the sample voltage and $V_{m}$ the voltage modulation amplitude. Six Lorentzians are used to model two YSR peaks and four molecular orbitals.

The overall fit (dashed red curve in Figure S6) reproduces the experimental spectrum (black curve).

The red and blue curves are attributed to LUSO and LUSO+1 located at (12.5 \pm 0.2$) \mathrm{meV}$ and $(47.3 \pm 1.1) \mathrm{meV}$, respectively, while the purple and green curves correspond to contributions 


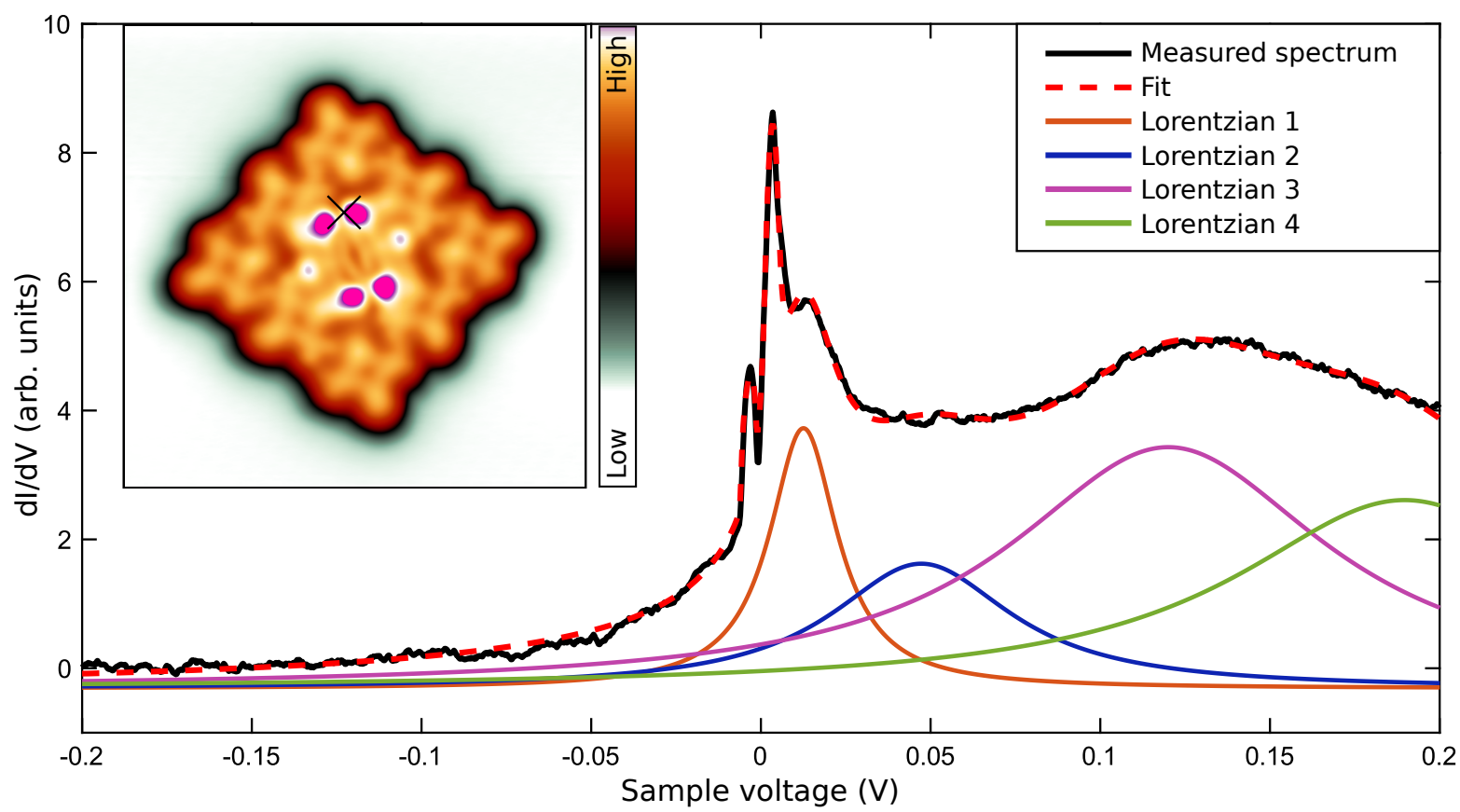

Figure S6: Identification of LUSO and estimation of charge transfer. Background-subtracted $d I / d V$ spectrum (black line) from the center of a supramolecule A shown in the topograph (width: $8 \mathrm{~nm}$ ) in the inset. The spectrum was measured at the position indicated by a cross (voltage modulation $2.5 \mathrm{mV}$, feedback loop opened at $I=500 \mathrm{pA}$ and $V=-200 \mathrm{mV}$ ). The red dashed line shows the sum of six Lorentzians convolved with the instrument response function $f_{\text {lock-in. Two }}$ Lorentzians, which are not shown, account for the YSR peaks. The other Lorentzians are displayed as solid lines. The resonance in orange, attributed to the LUSO, is centered at $(12.5 \pm 0.2) \mathrm{meV}$ and has a half width at half maximum $\Gamma=(12.2 \pm 0.6) \mathrm{meV}$.

from neighboring molecules. The difference conforms to an energy splitting of $35 \mathrm{meV}$ between the LUSO and LUSO+1, in line with the $47 \mathrm{meV}$ splitting observed for a single molecule.

The orange curve (Figure S6), associated to the LUSO, crosses the Fermi level. Approximately $25 \%$ of the peak area is below the Fermi level. As each orbital can accommodate two electrons, the position of the LUSO indicates a charge transfer of $\approx 0.5 \mathrm{e}$ from the substrate to the supramolecule.

\section{E. YSR resonances for additional tautomer configurations}

Figure S7 shows $d I / d V$ spectra of different tautomer configurations. These measurements are complementary to that shown in Figure 10 of the main manuscript. Although the datasets were acquired on different supramolecules and with different tip apices, the spectra can be simulated with the same parameters $\epsilon_{0}, \Gamma$ and $\Delta$, showing the reproducibility of the measurements. We nonetheless slightly modified the Gaussian broadening from a standard deviation of $120 \mu \mathrm{V}$ to $155 \mu \mathrm{V}$, which essentially reflects a small change in energy resolution.

The YSR energies and the asymmetries extracted from the fits to the experimental data are shown 
in Figures S7c-f (red points). For $\Gamma=18 \mathrm{meV}$ and $U=7 \mathrm{meV}$, the model predicts a particular evolution of $E_{\mathrm{YSR}}$ and $\chi$ as a function of the average energy of the spin-up and spin-down orbitals $\epsilon_{0}$ (solid black curves in Figs. S7). A small deviation from these values, e.g. $U=10 \mathrm{meV}$ or $\Gamma=30 \mathrm{meV}$, leads to a significant change in the evolution of $E_{\mathrm{YSR}}$ and $\chi$ (dashed black lines in Figs. S7c-f), which highlights the sensitivity of these parameters to the properties of the YSR resonances.
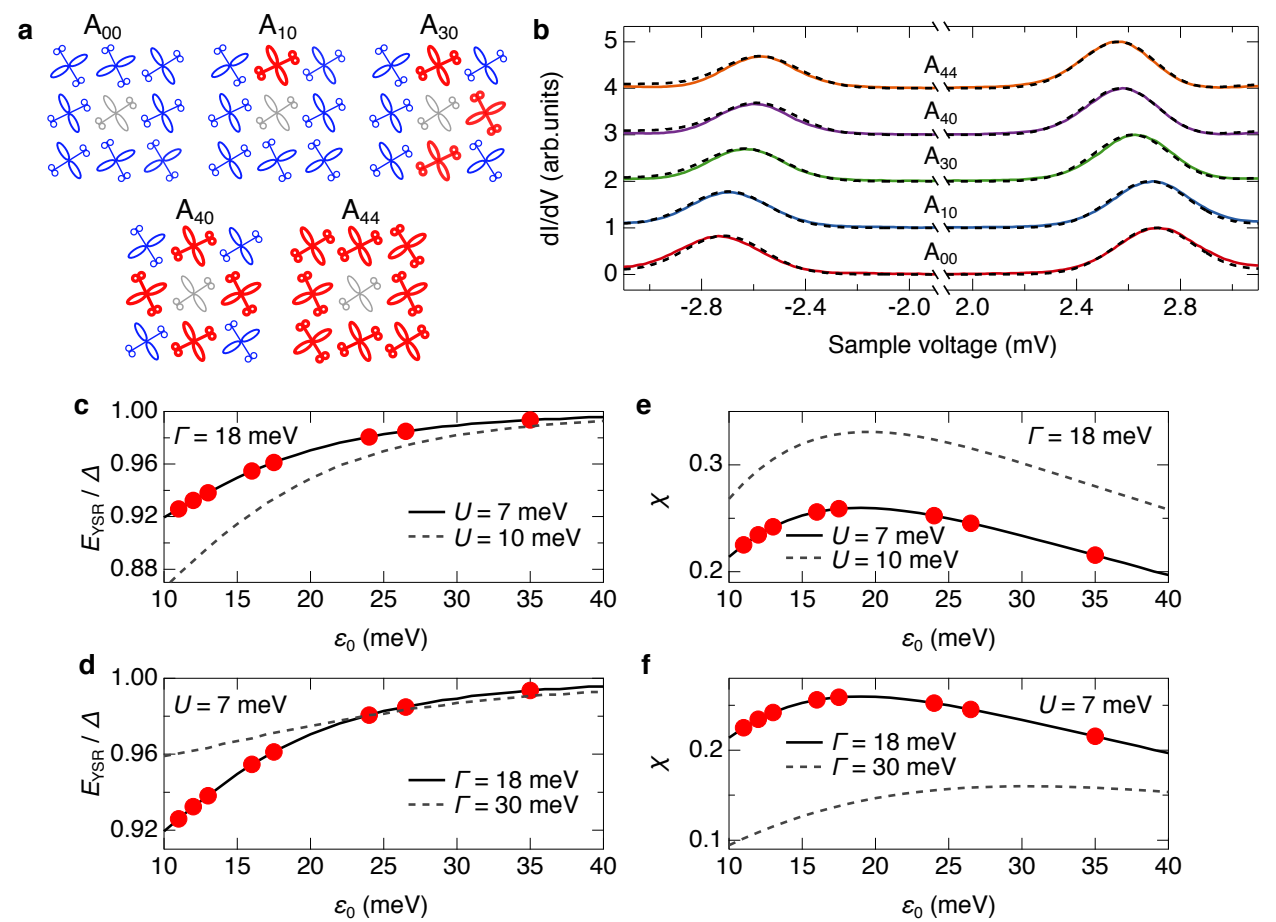

Figure S7: Fine tuning of YSR resonances by changing the tautomer configuration. a, Model of the tautomer configurations realized on a supramolecule. $\mathbf{b}$, Corresponding experimental $d I / d V$ spectra acquired on the central molecule (solid colored lines) along with simulated spectra (dashed black lines). The spectra were simulated as described in the caption to Figure 10 of the main manuscript using the same parameters with the exception of the standard deviation of the Gaussian broadening of $155 \mu \mathrm{V}$. LUSO energies for the different configurations are $\epsilon_{0} \in[11,12,16,26.5,35] \mathrm{meV}$. c and $\mathbf{d}$, Normalized YSR energy $E_{\mathrm{YSR}} / \Delta$ as a function of $\epsilon_{0}$ obtained with the model described in Ref. S4 for $U=7 \mathrm{meV}$ and $\Gamma=18 \mathrm{meV}$ (solid black curves). The red symbols show the experimentally determined ratio $E_{\mathrm{YSR}} / \Delta$ and $\epsilon_{0}$ (including data from Figure 10 of the main manuscript). The dashed black line in $\mathbf{c}(\mathbf{d})$ was calculated with $U=10 \mathrm{meV}$ and $\Gamma=18 \mathrm{meV}(U=7 \mathrm{meV}$ and $\Gamma=30 \mathrm{meV})$. e and $\mathbf{f}$, Peak-height asymmetry $\chi$ of the YSR resonance as a function of $\epsilon_{0}$ calculated using the parameters of $\mathbf{c}$ and $\mathbf{d}$. 


\section{Density functional theory related modeling}

We performed gas-phase density functional theory (DFT) calculations of individual molecules and supramolecules. We then employed an effective tight binding Hamiltonian to decompose the supramolecular orbitals into a combination of molecular orbitals. Based on the DFT and tightbinding analyses, we have identified several sources of intermolecular interaction. These sources of interaction are simulated in a simplified phenomenological model, which essentially reproduces the DFT and experimental results.

\section{A. DFT calculations}

DFT calculations were performed using the $\mathrm{PBE}^{\mathrm{S} 5}$ functional and the def $2 \mathrm{svp}^{\mathrm{S} 6}$ basis set using Gaussian 09. First, the structure of a single $\mathrm{H}_{2} \mathrm{Pc}$ molecule in the gas phase was optimized using Grimme's description of dispersion forces. ${ }^{\mathrm{S} 7}$ The input file for the supramolecular cluster calculations was constructed by placing 9 single molecules, each of them with the structure obtained from the single-molecule calculation, as depicted in Figure 11 of the main manuscript. The orientations of the two groups of molecules defined in Figure 11 as well as the tautomer configurations were adjusted to the experimental results of the considered supramolecule. The DFT calculation was then performed for the supramolecule without further structure optimization.

\section{B. Simulation of $\mathbf{d I} / \mathrm{dV}$ maps}

The simulated $d I / d V$ maps shown in the manuscript correspond to the transition matrix element $M$ squared evaluated for different positions $\left(x_{t i p}, y_{t i p}\right)$ of the tip using a modified Bardeen approach: ${ }^{\mathrm{s} 8}$

$$
M\left(x_{t i p}, y_{t i p}\right)=\frac{\hbar^{2}}{2 m} \int_{\Sigma}\left(\psi_{\text {tip }}^{*} \nabla \psi_{\text {sample }}-\psi_{\text {sample }} \nabla \psi_{\text {tip }}^{*}\right) d S
$$

where $\hbar$ and $m$ are the reduced Planck constant and the electron mass. The integral is carried out over a plane $\Sigma$ between the tip and the molecule. The wave function of the tip $\psi_{\text {tip }}$ is assumed to be spherical:

$$
\psi_{\text {tip }}=\frac{a}{r} \exp (-k r),
$$

where $a$ is a constant, $k=\sqrt{2 m \phi} / \hbar$ ( $\phi$ is the work function of the tip), $r$ the distance between the tip apex at $\left(x_{t i p}, y_{t i p}, z_{t i p}\right)$ and a point of interest in space $(x, y, z)$. In our calculations, $\psi_{\text {sample }}$ is substituted by the wave function of the molecular orbitals of interest (e.g. LUMO). 


\section{Effective tight binding Hamiltonian from DFT}

The DFT calculations of the single molecules (supramolecules) provide Kohn-Sham energies $\epsilon_{i}$ $\left(E_{j}\right)$ and wave functions $\phi_{i}\left(\Psi_{j}\right)$, which are represented in a localized basis of atomic orbitals. The indices $j$ and $i$ respectively enumerate the (supra-) molecular orbitals (MO). Both sets of $\phi_{i}$ and $\Psi_{j}$ orbitals are orthonormal:

$$
\sum_{x} \phi_{i}^{*}(x) \phi_{i^{\prime}}(x)=\delta_{i i^{\prime}}, \quad \sum_{x} \Psi_{j}^{*}(x) \Psi_{j^{\prime}}(x)=\delta_{j j^{\prime}}
$$

where $x$ is a combined position and atomic-orbital coordinate. The supramolecular wave functions can be expressed as a linear combination of single molecular wave functions. The coefficients are obtained by projection:

$$
V_{(k, i), j}=\sum_{x}\left[\hat{R}_{k} \phi_{i}^{*}(x)\right] \Psi_{j}(x),
$$

where the matrix $\hat{R}_{k}$ shifts and rotates the states of the single molecules, so that the atom positions coincide with those of molecule $k \in[1,9]$ in the supramolecule. In the analysis below, we used $i \in$ \{HOMO, LUMO, LUMO+1 $\}$ to expand the 9 highest occupied and 18 lowest unoccupied supramolecular orbitals, resulting in $\hat{V}$ being a $27 \times 27$ matrix. This subset of $i$ represents an almost complete basis set (for the subspace of interest), as $\sum_{k, i}\left|V_{(k, i), j}\right|^{2}>0.99$ for all $j$, and is therefore expected to reliably describe the supramolecular DFT solutions of interest. The considered tight-binding Hamiltonian $H$ in a single $\mathrm{MO}$ basis reads:

$$
\hat{H}=\hat{V}^{\dagger} \hat{D} \hat{V}
$$

where $\hat{D}$ is a matrix containing the energies $E_{j}$ on its diagonal. The matrix $\hat{H}$ contains on-site energies (diagonal elements) and hopping matrix elements (off-diagonal elements). The former ones are hereafter expressed relative to the energies $\epsilon_{i}$ of the unperturbed MO and therefore represent the molecule-dependent energy changes of the considered MO $i$ due to the interaction with neighbors.

The obtained matrix $\hat{H}$ is illustrated for two configurations of a supramolecule, namely $\mathrm{A}_{44}$ and $\mathrm{A}_{00}$ that respectively exhibit the maximum and minimum energy shift of the central molecule LUMO. In the $\mathrm{A}_{44}$ configuration, the HOMO, LUMO and LUMO+1 of the central molecule are all shifted by approximately $-200 \mathrm{meV}$ (numbers in red panels in Figure S8). The energy shifts of the HOMO, LUMO and LUMO+1 are virtually identical for each molecule in the cluster, which suggests an electrostatic origin. In contrast, the hopping matrix elements (numbers in blue panels in Figure S8) vary with the molecular orbitals involved and depend on their relative symmetry. 
However, the overall magnitude of the off-diagonal elements is small $(<1.5 \mathrm{meV})$ compared to the shift of the on-site energy (up to $-200 \mathrm{meV}$ ). From this we conclude that the hybridization between the molecules is negligible and that the shift of the LUMO on the central molecule mainly stems from the electrostatic stray field of the neighboring molecules. It should be noted that there are also non-zero matrix elements up to $16 \mathrm{meV}$ between the HOMO and the LUMO or LUMO+1 on the same molecule. Due to the large energy difference between the HOMO and the LUMO $\left(\epsilon_{\mathrm{LUMO}}-\epsilon_{\mathrm{HOMO}} \approx 1.4 \mathrm{eV}\right)$, these intramolecular inter-orbital matrix elements cause a negligible change of $E_{j}(<0.2 \mathrm{meV})$ and an admixture of $\approx 1 \%$ of $\phi_{\mathrm{HOMO}}$ to the unoccupied supramolecular states. However, these matrix elements contribute to an induced electrostatic dipole moment, which is absent from the isolated molecule.

Similar observations are made for the $\mathrm{A}_{00}$ configuration as shown in Figure $\mathrm{S} 9$. The on-site energies, however, are different. For instance, the energy shift of the central molecule MO is approximately $-115 \mathrm{meV}$ for $\mathrm{A}_{00}$ in contrast to $-200 \mathrm{meV}$ observed for configuration $\mathrm{A}_{44}$. This difference can be rationalized by different stray fields acting on the central molecule as detailed in Section D. In summary, the tight-binding projection shows that the DFT wave functions of the supramolecule are essentially single molecular wave functions rigidly shifted in energy. This shift depends on the position of a molecule inside the cluster and the tautomer configuration of the surrounding molecules.
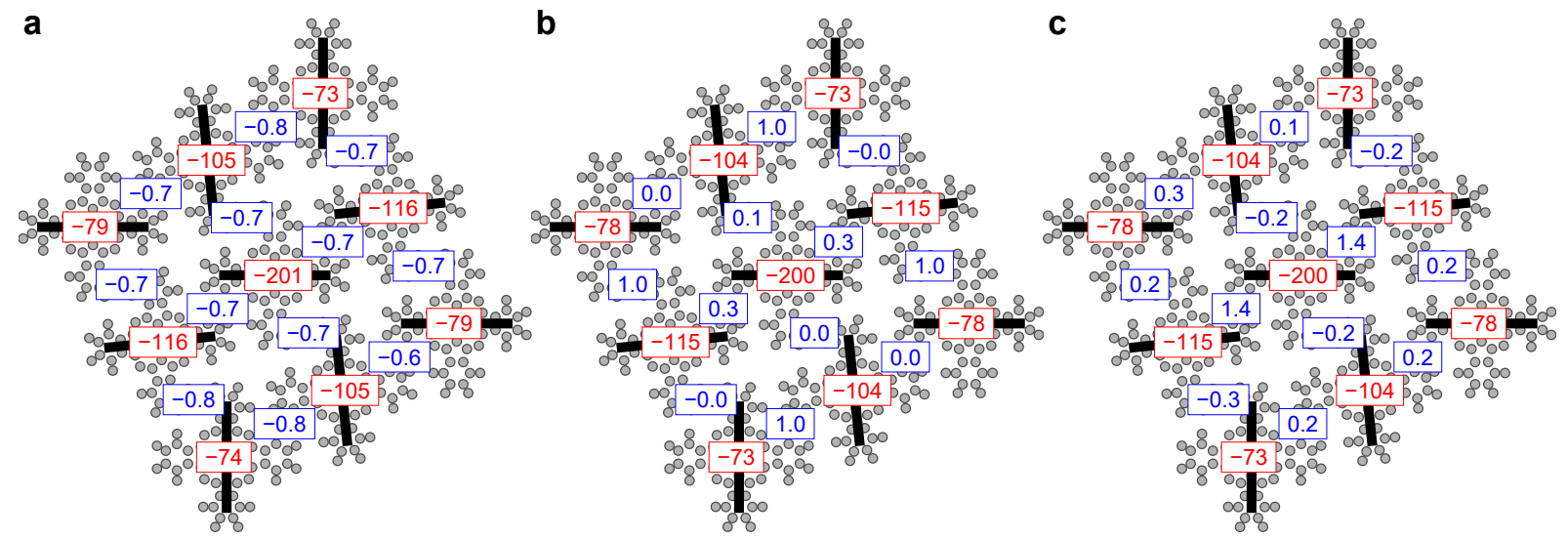

Figure S8: Tight-binding parameters (in $\mathrm{meV}$ ) of the $\mathrm{A}_{44}$ cluster. Gray disks represent atoms of the $\mathrm{H}_{2} \mathrm{Pc}$ molecules. On-site energies (red) of a, HOMO, b, LUMO and c, LUMO+1 relative to the respective energies of the isolated molecule. The hopping matrix elements of the same MO between neighboring molecules are indicated in blue. Black bars indicate the axes defined by the pyrrolic hydrogen atoms. 

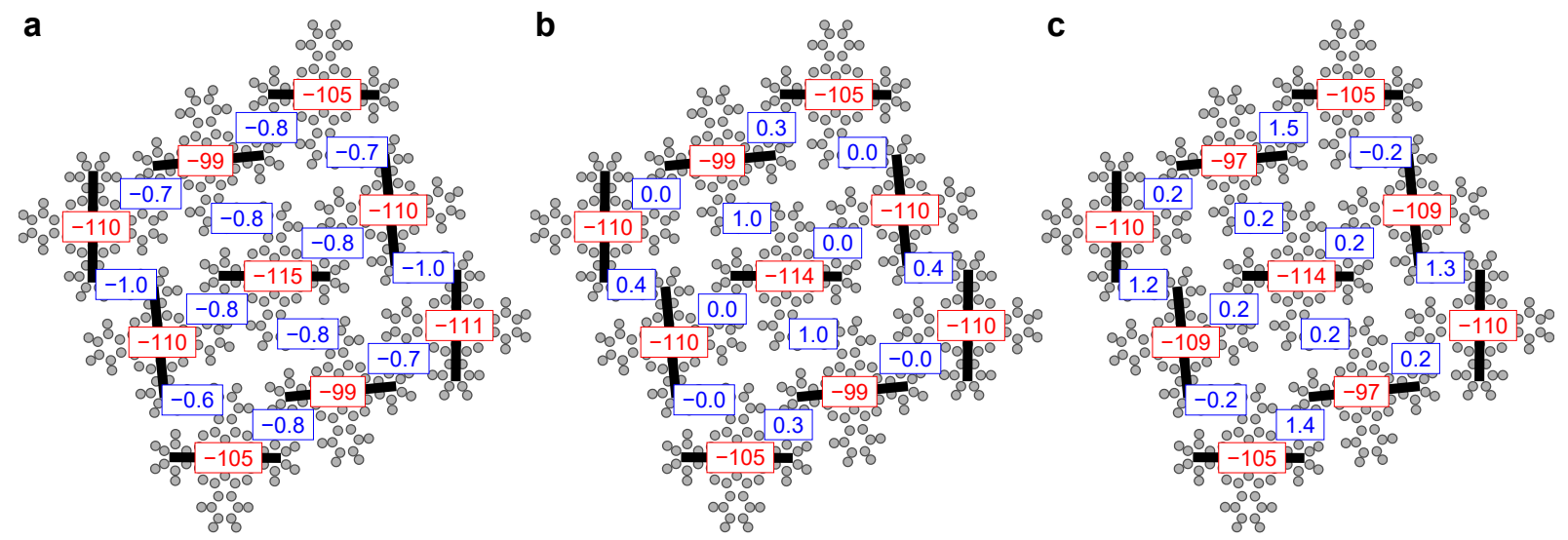

Figure S9: Tight-binding parameters (in meV) of the $\mathrm{A}_{00}$ cluster. Same as Figure S8 but for tautomer configuration $\mathrm{A}_{00}$, which causes the smallest reduction of the on-site energy on the central molecule.

\section{Phenomenological intermolecular interaction model}

The tight-binding projections indicate a rigid energy shift of all orbitals on a molecule, that may be due to an electrostatic interaction. The charge distribution, as calculated by DFT, is analyzed in the following. While the single molecule is neutral, polar bonds inside the molecule produce an electrostatic stray field with a quadrupole moment in lowest order of the multipole expansion. The quadrupole field is linked to the positions of the pyrrolic hydrogen atoms and can be inverted upon tautomerization. Furthermore, inside the cluster, the electrostatic field of neighboring molecules induces an electrostatic polarization by charge redistribution. Below, we reproduce the energy shifts of all molecules obtained from the tight-binding projections by the stray fields originating from DFT-derived static and induced charges. The electrostatic screening by the electrons of the substrate, absent in the DFT calculations, is accounted for in the model by including image charges. This leads to a good agreement between the interaction model and LUMO energies extracted from the experiments.

\section{Description of the model}

We express the LUMO energy $E_{i}$ of molecule $i$ within a cluster as

$$
E_{i}(\vec{t})=E^{\text {single }}+Q_{i}(\vec{t})+P_{i}(\vec{t})+V_{i}
$$

The numbering scheme of the molecules is shown in Figure 5 of the main manuscript. The tautomer configuration is described by a vector $\vec{t}$. $E^{\text {single }}$ is the LUMO energy of an isolated $\mathrm{H}_{2} \mathrm{Pc}$ molecule. $Q_{i}$ accounts for the quadrupole-like electrostatic potential generated by unperturbed $\mathrm{H}_{2} \mathrm{Pc}$ molecules and is directly linked to their tautomer configuration. $P_{i}$ takes into account the 


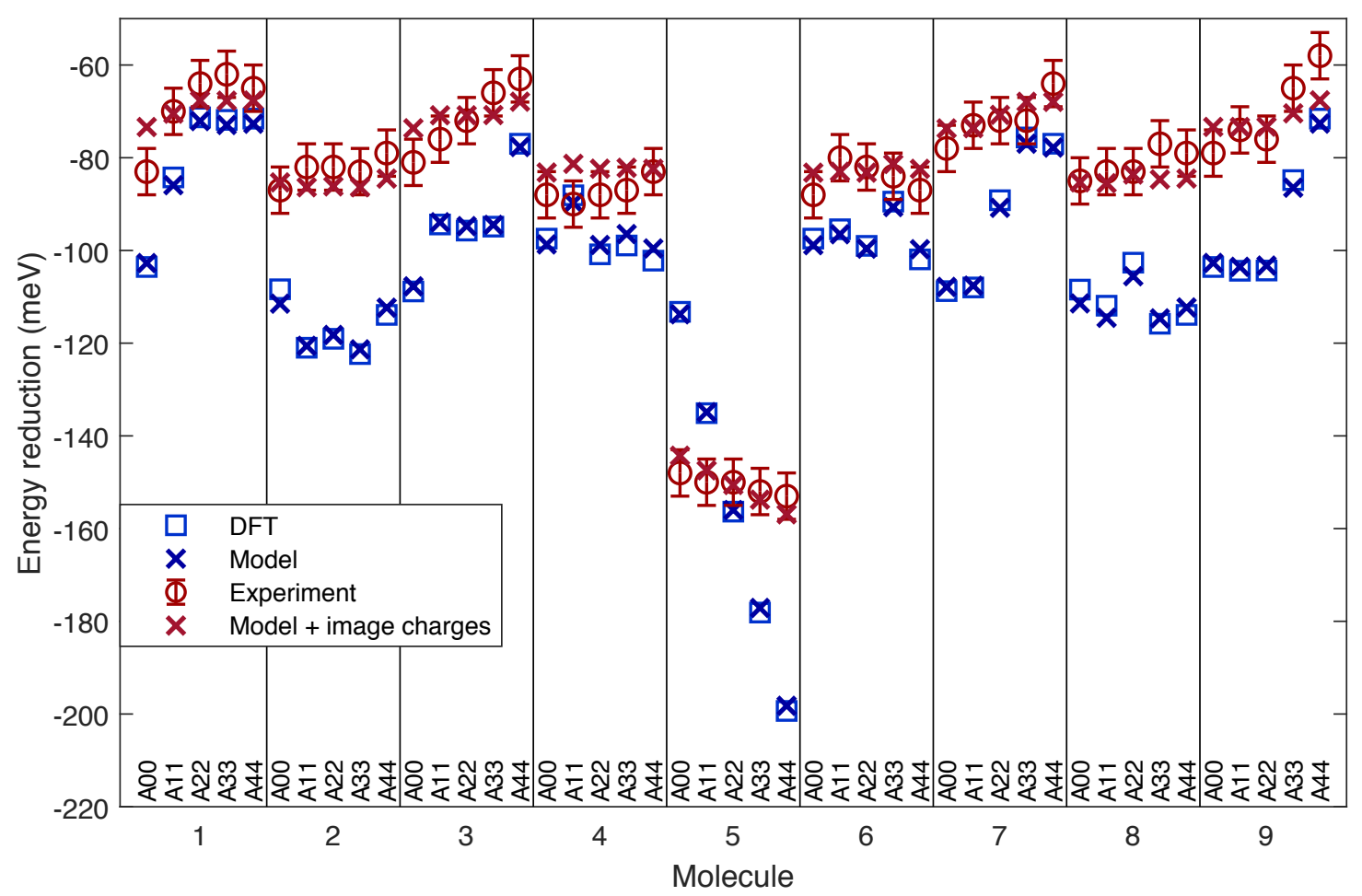

Figure S10: Reduction of LUMO energy due to intermolecular interaction. The LUMO energy reduction is shown for each of the molecules (numbers 1 to 9) of type A cluster in five characteristic tautomer configurations $\left(\mathrm{A}_{00}\right.$ to $\left.\mathrm{A}_{44}\right)$. Blue squares, blue crosses, red circles, and red crosses show the results of gas-phase DFT calculations (Section C.), Equation 7, experiments (Figure 5 of the main manuscript), and Equation 7 including the effect of image charges in the substrate. The experimental data were extracted from $d I / d V$ spectra acquired over the centers of the respective molecules. It is assumed that the energy of vibrational excitation is identical for all molecules. An estimated uncertainty of $\pm 5 \mathrm{meV}$ of the experimental results is indicated. The following parameters have been used for the models. Blue crosses: $V_{\text {corner }}=-77 \mathrm{meV}$, $V_{\text {edge }}=-122 \mathrm{meV}, V_{\text {center }}=-152 \mathrm{meV}, k=0.82$. Red crosses: $V_{\text {corner }}=-66 \mathrm{meV}$, $V_{\text {edge }}=-85 \mathrm{meV}, V_{\text {center }}=-144 \mathrm{meV}, k=0.82, d_{\text {image }}=3.2 \AA$.

polarization of the molecules that leads to a small charge redistribution and further LUMO energy shifts. $V_{i}$ captures tautomerization-independent effects via three parameters:

$$
V_{i}= \begin{cases}V_{\text {corner }} & , i \in\{1,3,7,9\} \\ V_{\text {edge }} & , i \in\{2,4,6,8\} \\ V_{\text {center }} & , i=5 .\end{cases}
$$

The determination of the parameters is detailed in the subsections below. To test the reliability of the model, we compare estimates of the LUMO energies from the model with that from DFT cal- 

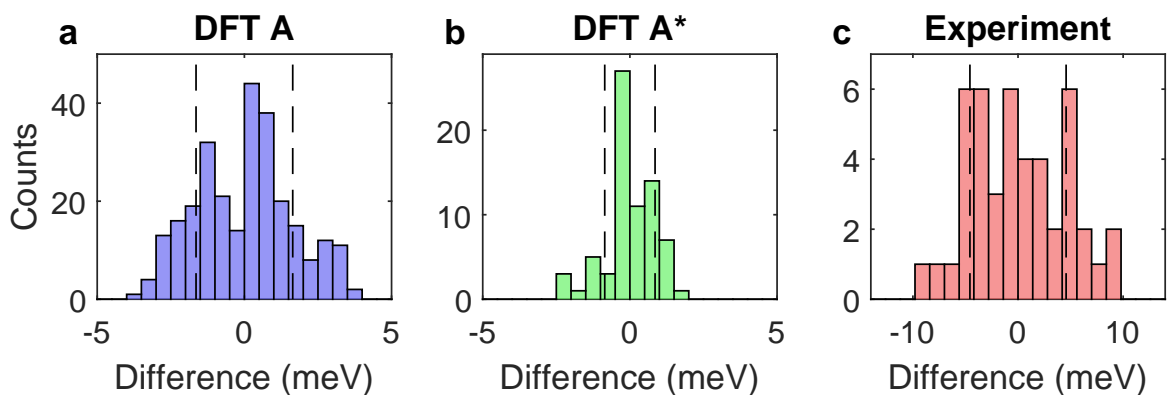

Figure S11: Reliability of LUMO energies from the model. Histograms of the deviations of the LUMO energy reductions of the model from the DFT calculations and the experiments. $\mathbf{a}$ and $\mathbf{b}$, model vs. DFT for supramolecules A and $\mathrm{A}^{*}$. c, Model $v s$. experimental results on supramolecule A. The histograms are based on a 270, b 108 and $\mathbf{c} 45$ molecules. The model calculations in $\mathbf{c}$ take image charges into account. Dashed lines indicate differences of one standard deviation.

culations and experiments. The blue squares in Figure S10 show the LUMO energy reductions (see Section C.) for the nine molecules of a supramolecule A in five different tautomer configurations. Estimates of the corresponding energies, calculated using Equation 7, are shown as blue crosses. The agreement is quite good. The model essentially captures all the molecule- and configurationdependent energy reductions and the deviations from DFT results are limited to a few meV. We further tested the phenomenological model to DFT results from 30 different tautomer configurations of A-type clusters and 12 calculations for $\mathrm{A}^{*}$ enneamers. The energy differences between the predictions of the model and DFT are displayed with histograms in Figures S11a and b. For configuration $\mathrm{A}\left(\mathrm{A}^{*}\right)$, the maximum deviation is $4 \mathrm{meV}(2.5 \mathrm{meV})$ and the maximum relative difference (not shown) is $4.5 \%(2.5 \%)$. In summary, the model provides estimates for the LUMO energies despite its simplicity.

The experiments were carried out on a $\mathrm{Pb}(100)$ substrate. While DFT calculations and the model (blue squares and crosses in Figure S10) essentially reproduce the experimental molecule-tomolecule and the configuration-to-configuration energy reductions (red circles), there are some discrepancies. In particular for molecule 5, at the center of the supramolecule, the LUMO energy changes much less than the gas-phase DFT predictions. This is actually to be expected because electrostatic intermolecular interactions are to some extent screened on the metal substrate, which reduces the corresponding energy shifts. We take the screening into account by placing image charges in the substrate at a distance $d_{\text {image }}$ from the molecules and by allowing the $V_{i}$ to change. The best agreement between the model and the experimental data is found for $d_{\text {image }}=3.2 \AA$ (red crosses and circles in Figure $\mathrm{S} 10$ ). On the surface, $V_{\text {corner }}, V_{\text {edge }}$ and $V_{\text {center }}$ increase (i.e. are less negative) by 9,37 and $8 \mathrm{meV}$, respectively. The histogram in Figure S11c shows the deviation of the LUMO energy reduction between the model with image charges and experimental data from 45 molecules. The largest deviation is $9.6 \mathrm{meV}$ and the standard deviation reads $4.6 \mathrm{meV}$. In other 


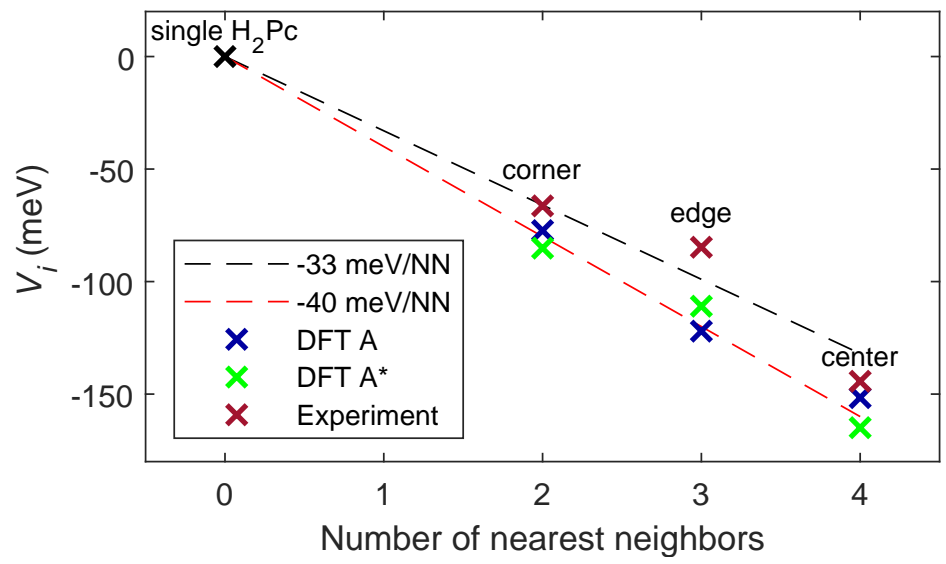

Figure S12: Nearest neighbor interaction. Parameters $V_{i}$ adjusted to fit DFT results of configurations $\mathrm{A}$ and $\mathrm{A}^{*}$ (blue and green crosses) and experimental results on cluster A (brown crosses) shown as a function of the number of nearest neighbors. The dashed lines exhibit slopes of $-33 \mathrm{meV} / \mathrm{NN}$ (black) and $-40 \mathrm{meV} / \mathrm{NN}$ (red).

words, the model predicts the energy reductions with a error margin of $\approx 10 \%$. This agreement suggests that the energy contributions discussed for the gas phase clusters are still present on the surface albeit reduced by screening.

\section{Nearest neighbor interaction}

The variable $V_{i}$ was adjusted to better fit the DFT or the experimental results. We find that, to a good approximation, $V_{i}$ is proportional to the number of nearest neighbors (NN) and hence reckon that it originates from a nearest neighbor interaction (Figure S12). This interaction significantly reduces the LUMO energy of the considered molecule by 33 to $40 \mathrm{meV}$ per nearest neighbor.

\section{Electrostatic interaction from unperturbed $\mathrm{H}_{2}$ Pc molecules}

As described in the main manuscript, the inhomogeneous charge distribution of $\mathrm{H}_{2} \mathrm{Pc}$ leads to a sizable electrostatic potential $\Phi$. For a molecule $i$, the sum of the electrostatic potentials of the neighbors changes the LUMO energy by:

$$
Q_{i}=-k \int_{V} d \vec{r} \cdot\left|\Psi_{i}(\vec{r})\right|^{2} \cdot \sum_{n \neq i} \Phi_{n}(\vec{r})
$$

where $\Phi_{n}$ is the electrostatic potential induced by the unperturbed molecule $n,\left|\Psi_{i}(\vec{r})\right|^{2}$ is the LUMO electron density of molecule $i$, and $V$ the volume of integration. The factor $k$ scales the electrostatic interaction. It takes for instance into account that the molecule of interest $i$ partially screens the electrostatic potential generated by its neighbors. In the following, the value is fixed to 
$k=0.82$, which leads to the best agreement.

By evaluating Equation 9 for all tautomer configurations we construct the matrix $\hat{Q}$. The element $Q_{i, j}$ is half of the LUMO energy change of molecule $i$ upon tautomerization of molecule $j$. The vector $\vec{Q}$ describes the energy changes of the nine molecules of a cluster with tautomer configuration $\vec{t}$ :

$$
\vec{Q}(\vec{t})=\frac{1}{2}\left(\vec{Q}_{\mathrm{T}_{00}}+\vec{Q}_{\mathrm{T}_{44}}\right)+\hat{Q} \cdot \vec{t}
$$

$\vec{t}$ is a nine-component vector with the $t_{i} \in\{-1,1\}$ indicating the orientation of the pyrrolic hy-

drogen atoms in molecule $i . \vec{Q}_{\mathrm{T}_{00}}\left(\vec{Q}_{\mathrm{T}_{44}}\right)$ with $\mathrm{T} \in\left\{\mathrm{A}, \mathrm{A}^{*}\right\}$ is the LUMO energy reduction in the configuration for which none of (all) the axes through the pyrrolic hydrogen atoms pass through the central molecule, which corresponds to $t_{i}=-1\left(t_{i}=1\right)$ for all molecules.

\section{Electrostatic interaction due to charge redistribution}

The polarizability of the molecules and the overlap of the wave functions lead to a charge redistribution over the supramolecule. Figure S13 shows the induced charge difference on every atom of the molecules for a cluster A and $\mathrm{A}^{*}$. The largest changes occur on edge (corner and central) molecules at peripheral $\mathrm{C}$ and $\mathrm{H}$ atoms for type $\mathrm{A}\left(\mathrm{A}^{*}\right)$ cluster and lead to the formation of dipoles pointing toward neighboring molecules. Interestingly, for cluster type A, the dipoles point toward the central molecule thereby decreasing the corresponding LUMO energy. In contrast, the induced dipoles lead to a LUMO energy increase of the central molecule for cluster type $\mathrm{A}^{*}$. The orientation of the dipoles is consistent with the presence and absence of YSR resonances for supramolecules $\mathrm{A}$ and $\mathrm{A}^{*}$, respectively.

The change in the electrostatic potential, due to the charge redistribution, is computed using Equation 9, where the potentials $\Phi_{n}$ result from the change in charges (Figure S13) instead of the static charges. In analogy to $\vec{Q}$, the vector $\vec{P}$ that describes the LUMO energy changes due to charge redistribution reads

$$
\vec{P}(\vec{t})=\frac{1}{2}\left(\vec{P}_{\mathrm{T}_{00}}+\vec{P}_{\mathrm{T}_{44}}\right)+\hat{P} \cdot \vec{t}
$$

\section{Discussion}

Figure S14 shows a representation of the matrices $\hat{Q}$ and $\hat{P}$ for a supramolecule A. The central coarse compartment indicates the impact of the tautomerization of a molecule $j$ on the LUMO energy of molecule 5. The largest contributions $Q_{5 j}$ are observed for $j=2,4,6,8$, i.e. the nearest neighbors of molecule 5. For $P_{5 j}$, tautomerization of the corner molecules induces the largest shifts, i.e. tautomerization of the next-nearest neighbors. We recall that $\hat{P}$ accounts for induced charges due to the polarizability of the molecules. The largest effect of the next nearest neighbors may be rationalized as follow. The tautomerization of a corner molecule affects the induced charges 

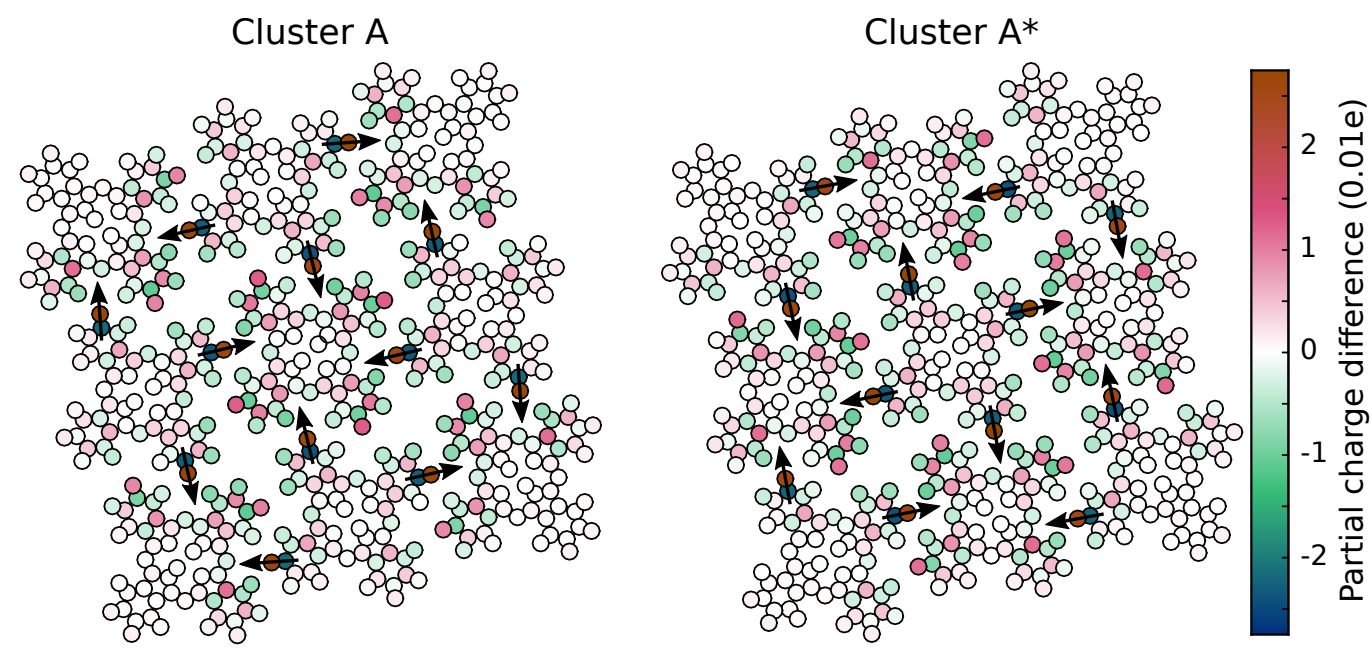

Figure S13: Charge redistribution upon assembly. The black circles represent atoms of the $\mathrm{H}_{2} \mathrm{Pc}$ molecules and the color within the circles encode the partial charge difference (see colorscale). The partial charge differences are obtained from the Mulliken charges of the supramolecules to which the charges of unperturbed $\mathrm{H}_{2} \mathrm{Pc}$ is subtracted for each molecule. The largest difference is found on the edge (central and corner) molecules in cluster $\mathrm{A}_{44}\left(\mathrm{~A}_{44}^{*}\right)$, where the respective $\mathrm{C}$ and $\mathrm{H}$ atoms form dipoles (arrows).

on its neighbors, i.e. edge molecules. In turn, the induced charges of the edge molecules modify the electrostatic potential acting on the central molecule. The importance of nearest and next nearest neighbors for respectively $\hat{Q}$ and $\hat{P}$ is observed for all molecules $i$. The chirality of the supramolecules (Figure 11 of the main manuscript) reduces the symmetry of matrices $\hat{Q}$ and $\hat{P}$. For instance, tautomerization of molecule $j=1$ increases the LUMO energy of molecule $i=4$ (see reddish square in the first column and fourth row in Figure S14a), while that of $j=7$ decreases it (green square, first column, sixth row), although both $j$ molecules are located at corners and are nearest neighbors of $i$.

Table S1 shows the amplitudes of the different contributions (including image charges) to the LUMO energy of the central molecule for four configurations of a supramolecule. The largest contribution arises from $V_{5}$, which depends on the number of nearest neighbors. This explains why the central molecule of a cluster systematically experiences the largest energy shift, possibly accompanied by YSR resonances when the shift is sufficient to cross the Fermi level.

The change of the tautomer configuration from $\mathrm{A}_{00}$ to $\mathrm{A}_{44}$ leads to a decrease of the LUMO energy by $13 \mathrm{meV}$. The decrease is driven by $Q_{5}\left(-15 \mathrm{meV}\right.$ variation), while the change in $P_{5}$ is in the other direction and tends to increase the LUMO energy. Therefore, the changes in LUMO and YSR energies for cluster type A discussed in the main text are essentially dictated by $Q_{5}$ and hence by the quadrupole field of the unperturbed neighbors.

Figure S13 shows that the induced charges within the supramolecule lead to dipoles. For cluster 

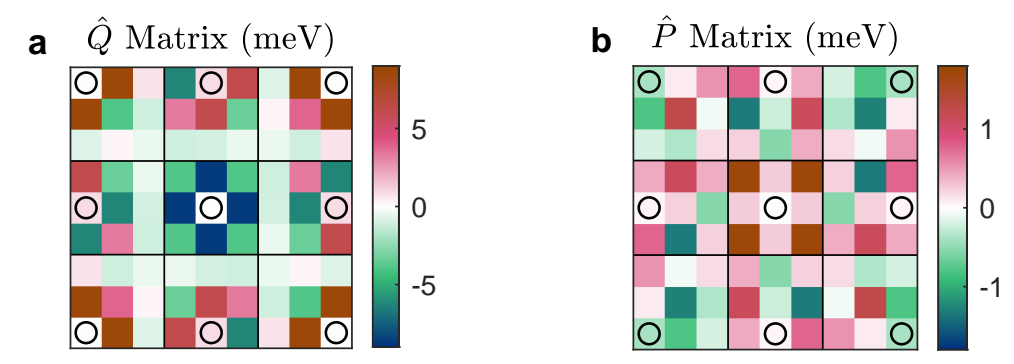

Figure S14: Electrostatic-interaction matrices $\hat{Q}$ and $\hat{P}$ for type A cluster (without image charges). The color scale encodes the values $\mathbf{a}, Q_{i}$ and $\mathbf{b}, P_{i, j}$, which correspond to half of the LUMO energy variation of molecule $i$ caused by the tautomerization of molecule $j$. The coarse compartments (surrounded by a thick solid line) focus on the entries affecting the energy of a given molecule $i$. The numbering scheme goes from left to right and top to bottom. For instance, the upper-right compartment, indicates the contributions on molecule 3. Within a compartment, the 9 squares distinguish the molecules $j$, with the same numbering scheme. The squares with $i=j$ are marked with a black circle. As an example, the brown square in the first column and second row in a indicates that the tautomerization of molecule $j=4$ leads to an increase of the molecule $i=1$ LUMO energy by $\approx 2 \times 8.6 \mathrm{meV}$ due to the change in the quadrupole-like field.

Table S1: LUMO energy shifts (in $\mathrm{meV}$ ) of the central molecule inferred from the model with image charges. $Q_{5}, P_{5}$ and $V_{5}$ denote the energy shifts on molecule 5 due to the quadrupolelike field of the unperturbed neighbors, the induced charges and the nearest neighbor interaction, respectively. $\Delta E_{5}=Q_{5}+P_{5}+V_{5}$ correspond to the total energy shift. $Q_{5}$ and $P_{5}$ were evaluated using Equations 10 and 11 (including image charges), respectively. Note that we do not have enough experimental data to accurately estimate $V_{5}$ for the model involving image charges.

\begin{tabular}{c|c|c|c|c} 
& $Q_{5}$ & $P_{5}$ & $V_{5}$ & $\Delta E_{5}$ \\
\hline $\mathrm{A}_{00}$ & 5 & -5 & -144 & -144 \\
$\mathrm{~A}_{44}$ & -10 & -3 & -144 & -157 \\
$\mathrm{~A}_{00}^{*}$ & 4 & 2 & - & - \\
$\mathrm{A}_{44}^{*}$ & -6 & 4 & - & -
\end{tabular}

type A, the nearest neighbors of the central molecule exhibit dipoles pointing toward the center. This leads to an energy decrease of the central molecule by approximately $5 \mathrm{meV}$ ( $P_{5}$ value in Table S1), taking into account the screening of the field by the substrate. In contrast, for type $\mathrm{A}^{*}$ supramolecules, the dipoles are located on the central molecule and point toward its neighbors. Accordingly, this shifts the LUMO energy of the central molecule upwards (positive $P_{5}$ value in Table S1). 


\section{References}

(S1) Li, C.; Wang, Z.; Lu, Y.; Liu, X.; Wang, L. Conformation-Based Signal Transfer and Processing at the Single-Molecule Level. Nat. Nanotechnol. 2017, 12, 1071-1076.

(S2) Pavliček, N.; Swart, I.; Niedenführ, J.; Meyer, G.; Repp, J. Symmetry Dependence of Vibration-Assisted Tunneling. Phys. Rev. Lett. 2013, 110, 136101.

(S3) Gruber, M.; Weismann, A.; Berndt, R. The Kondo Resonance Line Shape in Scanning Tunnelling Spectroscopy: Instrumental Aspects. J. Phys. Condens. Matter. 2018, 30, 424001.

(S4) Weismann, A.; Homberg, J.; Gruber, M.; Berndt, R. Anderson Impurity Model Parameters from Spectroscopy of Impurities on Superconductors. Unpublished.

(S5) Perdew, J. P.; Burke, K.; Ernzerhof, M. Generalized Gradient Approximation Made Simple. Phys. Rev. Lett. 1996, 77, 3865-3868.

(S6) Weigend, F.; Ahlrichs, R. Balanced Basis Sets of Split Valence, Triple Zeta Valence and Quadruple Zeta Valence Quality for H to Rn: Design and Assessment of Accuracy. Phys. Chem. Chem. Phys. 2005, 7, 3297-3305.

(S7) Grimme, S.; Antony, J.; Ehrlich, S.; Krieg, H. A Consistent and Accurate Ab Initio Parametrization of Density Functional Dispersion Correction (DFT-D) for the 94 Elements H-Pu. J. Chem. Phys. 2010, 132, 154104.

(S8) Chen, C. Introduction to Scanning Tunneling Microscopy; Oxford University Press: New York, 1993. 